\title{
Comparação Eletrocardiográfica entre Estimulação Cardíaca Artificial de Localização Apical, Septal e via de Saída do Ventrículo Direito
}

\author{
Electrocardiographic Comparison Between Artificial Cardiac Stimulation of \\ Apex, Septum and Right Ventricular Outflow Tract
}

Roberto Carlos Alvarez Coello ${ }^{1,2, *}$, Diego Patricio Serrano Piedra ${ }^{1,3,4}$

ORCID IDs

Coello RCA (D) https://orcid.org/0000-0002-2066-2410

Piedra DPS (D) https://orcid.org/0000-0001-6931-9069

\begin{abstract}
RESUMO
Contexto: A estimulação ventricular é prejudicial para a função cardíaca, com efeitos deletérios a longo prazo, alargando o complexo QRS, de modo que o melhor local de estimulação cardíaca ainda está sendo procurado. Objetivo: Comparar por medidas eletrocardiográficas a duração dos complexos QRS nos diferentes locais de estimulação cardíaca, dos prontuários de pacientes portadores de marcapasso que compareceram à consulta de Estimulação Cardíaca do Hospital Monte Sinai em Cuenca-Equador. Métodos: Foram analisados, retrospectivamente, 323 pacientes, no período de janeiro 2011 a novembro de 2018, classificando-os por morfologia eletrocardiográfica em estimulação apical, septal baixa, septal média e via de saída do ventrículo direito (VSVD). A duração dos complexos QRS estimulados foi quantificada usando um software digital que permite tal medição e armazena todos os registros. Subsequentemente, a comparação das médias dos diferentes locais de estimulação foi realizada utilizando ANOVA. Resultados: A idade média foi de 73,6 anos; 56,03\% corresponderam ao sexo masculino; 49,84\% tiveram estimulação apical, 21,67\% septal média, 15,78\% VSVD, 12,69\% septal baixa. Ao final da mensuração dos complexos QRS, obteve-se média no ápice de 179,94 ms, no septo baixo de 172,56 ms, no septo médio de 153,89 ms e em VSVD de 171,66 ms. Ao comparar esses valores com a ANOVA de médias, a duração do QRS estimulado no septo médio foi menor do que os demais locais, sendo estatisticamente significante $(p<0,001)$. Conclusão: A estimulação cardíaca no septo médio é uma alternativa viável, o que resulta em menor duração do complexo QRS, portanto, melhor sincronização da contração miocárdica, evitando também os efeitos deletérios da estimulação apical já conhecidos.
\end{abstract}

PALAVRAS-CHAVE: Estimulação cardíaca artificial; Eletrocardiografia; Análise de variância.

\begin{abstract}
Background: Ventricular pacing is detrimental to cardiac function, with more deleterious effects when it is apical, widening the QRS complex, so the best cardiac pacing site is still being sought. Objective: To compare, by electrocardiographic measurements, the duration of QRS complexes at different cardiac stimulation sites, from the medical records of patients with pacemakers, who attended the external cardiac stimulation consultation at Monte Sinai Hospital in Cuenca-Ecuador. Methods: A total of 323 patients were retrospectively analyzed from January 2011 to November 2018, classifying them by electrocardiographic morphology as apical, low septal, middle septal and right ventricular outflow tract (RVOT) stimulation. The duration of stimulated QRS complexes was quantified using digital software that allows such measurement and stores all records. Subsequently, the comparison of the means of the different stimulation sites was performed using ANOVA. Results: The average age was 73.6 years; $56.03 \%$ corresponded to males; $49.84 \%$ had apical stimulation, $21.67 \%$ middle septal, 15.78\% RVOT, 12.69\% low septal. At the end of the QRS complex measurements, an average on apex of 179.94 ms, on low septum of $172.56 \mathrm{~ms}$, an average on middle septum of $153.89 \mathrm{~ms}$ and on RVOT 171.66 ms were obtained. When comparing these values with the average ANOVA, the duration of the QRS stimulated in the middle septum was shorter than the other sites, being statistically significant $(p<0.001)$. Conclusion: Cardiac pacing in the middle septum is a viable alternative, which results in shorter QRS complex duration, therefore, better synchronization of myocardial contraction, also avoiding the deleterious effects of apical stimulation.
\end{abstract}

KEYWORDS: Artificial cardiac stimulation; Electrocardiography; Analysis of variance.

1. Hospital Monte Sinaí - Área de Estimulação Cardíaca - Cuenca - Equador

2.Universidade de São Paulo - Hospital das Clínicas da Faculdade de Medicina - Instituto do Coração - São Paulo (SP) - Brasil

3. Hospital Santa Inés - Área de Eletrofisiologia - Cuenca - Equador

4. Hospital José Carrasco Arteaga - Área de Eletrofisiologia - Cuenca - Equador

*Autor correspondente: rcacoello22@gmail.com

Recebido: 18 Ago 2019 | Aceito: 19 Nov 2019

Editor Associado: José Mário Baggio Júnior 


\section{INTRODUÇÃO}

Sabe-se que a estimulação ventricular é prejudicial para a função cardíaca global, com consequências clínicas a longo prazo, de modo que o melhor local de estimulação cardíaca ainda está sendo investigado sem resultados conclusivos. A estimulação apical do ventrículo direito está associada a efeitos deletérios na função cardíaca. Em geral, quanto mais estreito o complexo QRS, melhor a função ventricular esquerda ${ }^{1}$. A estimulação do ventrículo direito promove inversão da sequência natural de ativação elétrica cardíaca, gerando um bloqueio artificial de ramo esquerdo, com complexo QRS alargado, preditor de insuficiência cardíaca em portadores de marcapasso definitivo, pois quando grande parte das células é contraída, outra parte ainda está relaxada prejudicando o trabalho de contração cardíaca, que causa perda da eficiência sistólica ${ }^{2,3}$.Pode desenvolver ou agravar a regurgitação mitral ${ }^{4,5}$, aumentar o risco de fibrilação atrial (FA), insuficiência cardíaca (IC) e aumentar a mortalidade ${ }^{6,7} \mathrm{em}$ pacientes com disfunção sistólica.

A necessidade de estimulação cardíaca artificial mais fisiológica é indiscutivel. Por quase 40 anos, desde o uso da estimulação cardíaca artificial, o ápice do ventrículo direito foi considerado como o local preferido para o implante de eletrodos ventriculares ${ }^{8}$. A preocupação com a avaliação dos danos causados pela estimulação desse sítio é relativamente recente.

No momento,um sítio de estimulação alternativo está sendo investigado para evitar os efeitos deletérios do ápice e torná-lo o mais fisiológico possível. Os estudos publicados até agora são poucos e muitos deles inconclusivos. Devido a não haver nenhum registro no Equador, o que não permite contrastar as informações atualmente disponíveis, propõe-se um estudo retrospectivo que compara eletrocardiograficamente a duração dos complexos QRS com a estimulação cardíaca artificial nas vias apical, septal baixa, septal média e a via de saída do ventrículo direito (VSVD). Além disso, a duração dos complexos QRS pré e pós-estimulação é comparada para verificar a diferença entre eles.

\section{MÉTODOS}

Foi realizado um estudo comparativo retrospectivo. A amostra contém um total de 338 pacientes portadores de marcapasso com eletrodo ventricular (independente da patologia), sendo excluídos 11 pacientes por não apresentarem os registros eletrocardiográficos necessários e 4 pacientes menores de 16 anos. Assim, a amostra final foi de 323 pacientes atendidos na consulta de estimulação cardíaca no Hospital Monte Sinai da Cidade de Cuenca-Equador, no período de janeiro de 2011 a novembro de 2018, e foram classificados segundo parâmetros morfológicos eletrocardiográficos apicais (complexo QRS negativo em D2,D3 e aVF, basicamente complexos QS), septal baixo (eixo elétrico superior, porém, com positividade inicial em D2, D3 e aVF mas com relação $\mathrm{R} / \mathrm{S}<1$ ), septal médio (complexos QRS isodifásicos ou com clara tendência ao isodifasismo nas derivações D2, D3 e aVF) e septo da via de saída do VD (eixo elétrico evidentemente inferior, com relação $\mathrm{R} / \mathrm{S}>1$ ).

Foram obtidos 161 pacientes com estimulação apical (49,84\%), 70 com septal média (21,67\%),51 com VSVD (15,78\%), 41 com septal baixa (12,69\%) (Fig. 1).

Uma vez classificada pela localização da estimulação ventricular, a duração dos complexos QRS pré-estimulação (de pacientes que não eram totalmente dependentes do marcapasso ou que tiveram um registro anterior com seu próprio ritmo) e pós-estimulação foi medida utilizando um módulo de aquisição eletrocardiográfica digital para computador (ECG PC, marca $\mathrm{TEB}^{\circledR}{ }^{\circledR}$, que mantém registros de todos os pacientes. Uma vez que os dados foram coletados, eles foram comparados, primeiro com o teste $t$ de duas amostras entre estimulação apical e septal (septo baixo e médio). Subsequentemente, todas as localizações foram comparadas por análise da variância das médias ANOVA, para saber qual é o local de estimulação mais fisiológico. Além disso, foi comparado pelo teste $\mathrm{t}$ pareado entre a duração do QRS prévio e posterior de cada localidade para avaliar sua diferença. Uma análise dos dados atípicos foi feita de acordo com a distribuição das amostras para corroborar que não houve erros na medição, de modo que esses dados foram submetidos a uma segunda medida, que coincidiu com a primeira (Fig. 2).

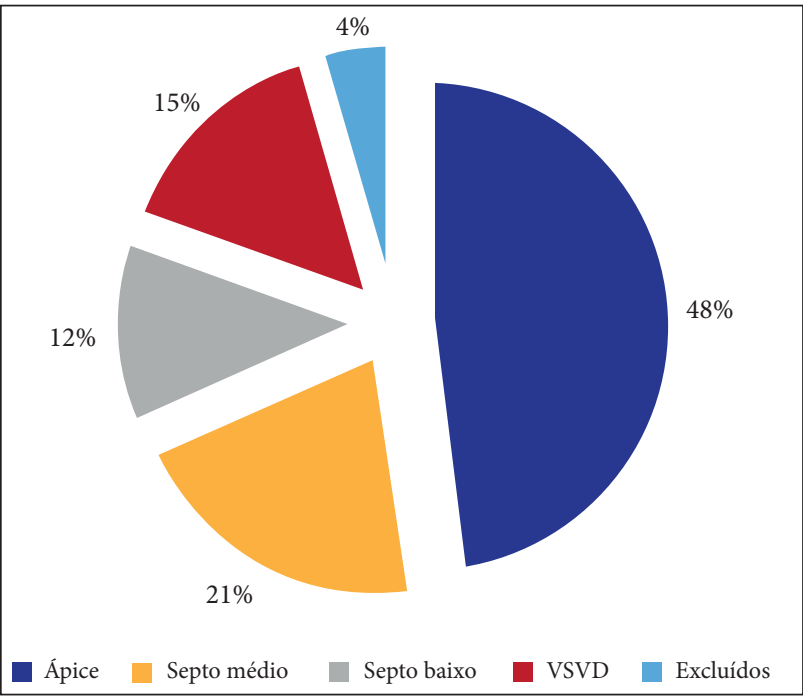

Figura 1. Distribuição da amostra. 
Um banco de dados foi projetado no software Microsoft Excel e posteriormente exportado para o Minitab, com o qual a análise estatística foi realizada.

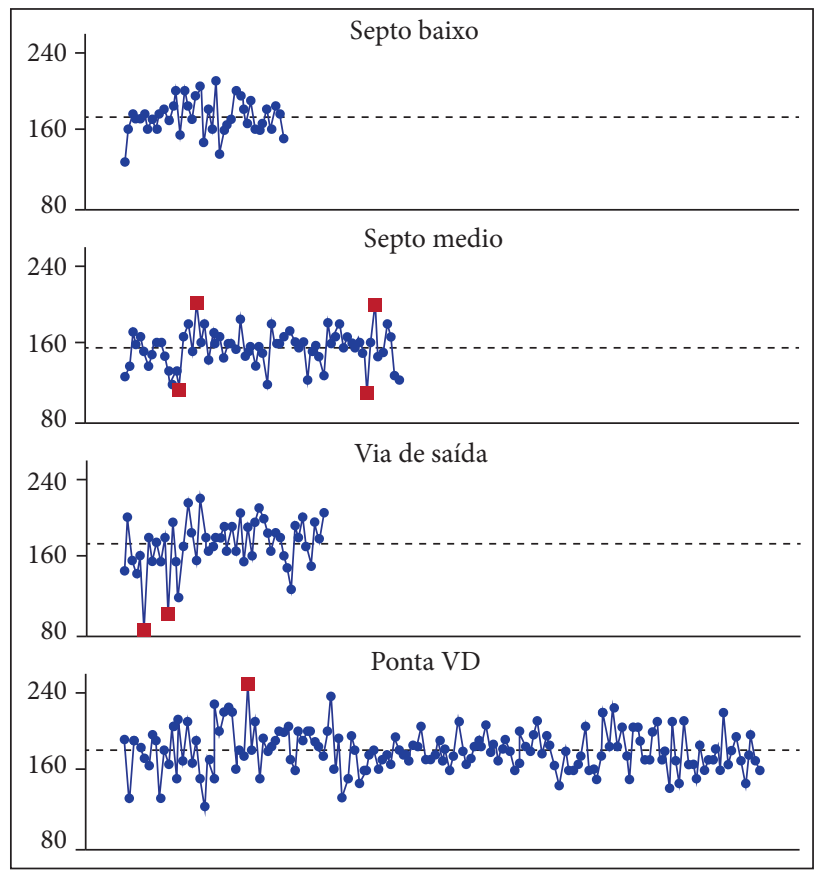

Figura 2. Distribuição dos dados pela localização, duração dos complexos QRS e localização dos dados atípicos (vermelho).

\section{RESULTADOS}

A idade média foi de 73,6 anos; 56,03\% corresponderam ao sexo masculino. Ao final da mensuração dos complexos QRS, obteve-se uma média no ápice de 179,94 ms com intervalo de confiança de $95 \%$ (IC) de $3,36 \mathrm{~ms}$ (176,55$183,32 \mathrm{~ms})$, no septo baixo de $172,56 \mathrm{~ms}$ IC $5,79 \mathrm{~ms}$ (166,77-178,35 ms), no septo médio $153,89 \mathrm{~ms}$ IC 4,70 ms (149,18 - 158,59 ms), e na VSVD 171,66 ms IC 7,71 ms (163,96-179,38 ms). Ao comparar a estimulação apical com a septal (septo baixo e médio) observa-se que a estimulação apical é maior $(\mathrm{p}<0,001)$.

Ao comparar as médias de todas as localizações por ANOVA, obtém-se que a estimulação do septo médio difere das demais, dando uma duração mais curta do complexo QRS, estatisticamente significante $(\mathrm{p}<0,001)$ (Tabela 1, Fig. 3).

Na comparação da duração dos complexos QRS pré e pós-estimulação, foram excluídos 69 pacientes totalmente dependentes do marcapasso e para os quais não havia registro prévio ao implante do marcapasso. A aplicação do teste $t$ pareado para cada um dos locais mostrou uma diferença estatisticamente significativa em todos
( $\mathrm{p}<0,001)$, indicando que após da estimulação, as médias de todos os locais aumentaram em duração (Fig. 4). Ao obter este resultado, os autores se perguntaram quanto os complexos QRS aumentam após da estimulação ventricular. Assim, a pré-estimulação média foi de $133,40 \mathrm{~ms}$ e a pós-estimulação foi de $167,30 \mathrm{~ms}$, resultando em um rango de $33,80 \mathrm{~ms}$.

Tabela 1. Comparação da duração das médias dos complexos QRS pela localização apical, septal e VSVD.

\begin{tabular}{ccccc}
\hline \multicolumn{5}{c}{ Localização da estimulação } \\
\hline Variável & Ápice & $\begin{array}{c}\text { Septo } \\
\text { baixo }\end{array}$ & $\begin{array}{c}\text { Septo } \\
\text { médio }\end{array}$ & VSVD \\
\hline $\mathbf{n}$ & 161 & 41 & 70 & 51 \\
$\begin{array}{c}\text { Média do } \\
\text { QRS (ms) }\end{array}$ & 179,94 & 172,56 & 153,88 & 171,66 \\
IC 95\% & $176,58-$ & $166,77-$ & $149,18-$ & $163,96-$ \\
& 183,30 & 178,35 & 158,59 & 179,38 \\
$\begin{array}{c}\text { Significância } \\
\text { estatística }\end{array}$ & \multicolumn{5}{c}{$\mathrm{p}<0,001$} \\
\hline
\end{tabular}

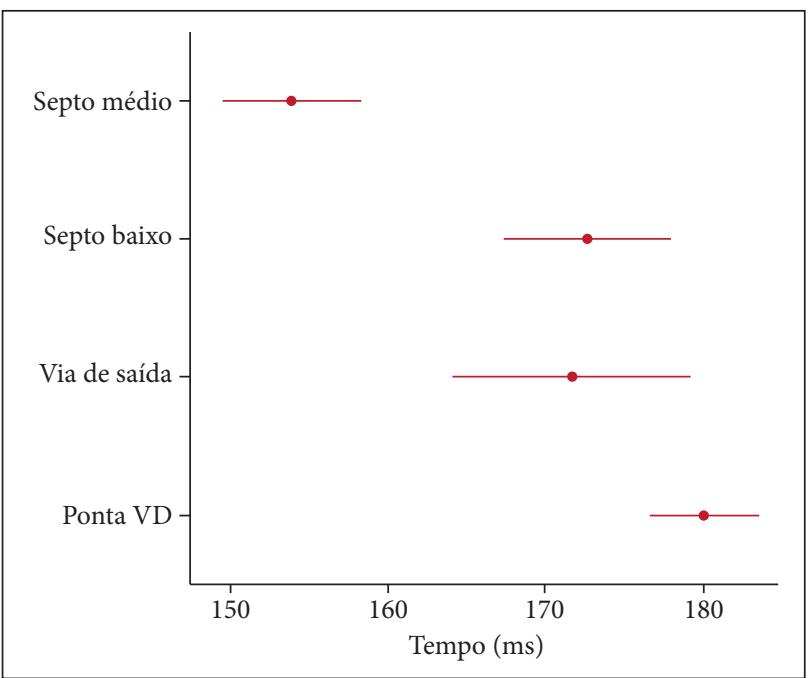

Figura 3. Comparação por ANOVA das médias dos complexos QRS com seus respectivos intervalos de confiança.

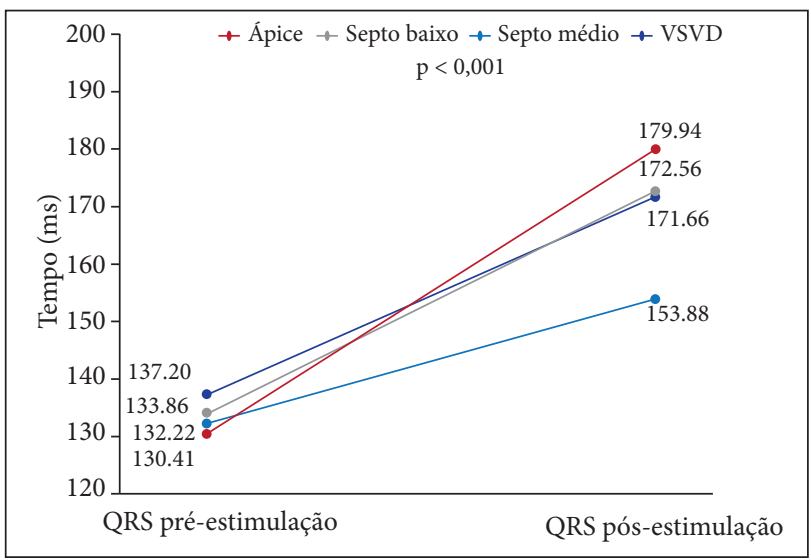

Figura 4. Comparação da duração em milissegundos dos complexos QRS pré e pós-estimulação das diferentes localizações. 


\section{DISCUSSÃO}

O sítio mais fisiológico da estimulação ventricular ainda é um desafio para a área da estimulação cardíaca. Há poucos estudos comparativos publicados com os quais contrastar a informação. Com os resultados obtidos, recomenda-se evitar (sempre que possível) a estimulação apical do VD e preferir a estimulação ao nível do septo interventricular médio, catalogada há anos como mais trabalhosa e tardia, porém na experiência dos autores deste trabalho, com a técnica apropriada e com auxílio de projeções oblíquas, não foi observada diferença com o implante apical, ao contrário, uma menor taxa de complicações no nível septal, principalmente perfurações. Sabe-se que ainda há muitas questões a serem resolvidas, por isso os autores recomendam fazer estudos prospectivos em que não só parâmetros eletrocardiográficos sejam medidos, mas também parâmetros clínicos, ecocardiográficos e laboratoriais.

\section{CONCLUSÕES}

Informações valiosas foram recebidas sobre a população. Foi demonstrado estatisticamente que a estimulação cardíaca artificial em geral aumenta a duração do complexo QRS com um rango de $33,8 \mathrm{~ms}$.
A estimulação apical prolonga mais a duração do QRS do que a septal. O septo médio de acordo com parâmetros eletrocardiográficos na amostra de pacientes utilizada é o melhor local de estimulação ventricular, pois tem duração dos complexos QRS significativamente menor que os demais locais, portanto, pode-se extrapolar que há uma melhor sincronização de contração miocárdica, mais próxima a fisiológica, além de evitar os efeitos deletérios já conhecidos da estimulação apical.

Por tudo isso, os autores consideram o septo interventricular médio uma alternativa viável e que deve ser tentada sempre que possível. Em contraste, o ápice é o local onde o complexo QRS é mais prolongado e, devido à evidência disponível no momento, um dos piores locais de estimulação.

\section{CONTRIBUIÇÃO DOS AUTORES}

Conceitualização, Alvarez Coello RC e Serrano Piedra DP; Metodologia, Alvarez Coello RC e Serrano Piedra DP; Investigação, Alvarez Coello RC; Análise Estatística, Alvarez Coello RC; Redação, Alvarez Coello RC e Serrano Piedra DP; Revisão, Serrano Piedra DP; Edição, Alvarez Coello RC e Serrano Piedra DP; Recursos, Alvarez Coello RC e Serrano Piedra DP.

\section{REFERÊNCIAS}

1. Xiao HB, Lee $\mathrm{CH}$, Gibson DG. Effect of left bundle branch block on diastolic function in dilated cardiomyopathy. Br Heart J. 1991;66(6):443-7. https://doi.org/10.1136/ hrt.66.6.443

2. Manolis AS. The deleterious consequences of right ventricular apical pacing: time to seek alternate site pacing. Pacing Clin Electrophysiol. 2006;29(3):298-315. https://doi. org/10.1111/j.1540-8159.2006.00338.x

3. Su Y, Pan W, Gong X, Cui J, Shu X, Ge J. Relationships between paced QRS duration and left cardiac structures and function. Acta Cardiol. 2009;64(2):231-8. https://doi.org/10.2143/ AC.64.2.2036143

4. Karpawich PP, Rabah R, Haas JE. Altered cardiac histology following apical right ventricular pacing in patients with congenital atrioventricular block. Pacing Clin Electrophysiol. 1999;22(9):1372-7. https://doi. org/10.1111/j.1540-8159.1999.tb00631.x
5. Hanna SR, Chung ES, Aurigemma GP, Meyer TE. Worsening of mitral regurgitation secondary to ventricular pacing. J Heart Valv Dis. 2000;9(2):273-5.

6. Skanes AC, Krahn AD, Yee R, Klein GJ, Connolly SJ, Kerr $C R$, et al. Progression to chronic atrial fibrillation after pacing: The Canadian Trial of Physiologic Pacing. J Am Coll Cardiol. 2001;38(1):167-72. https://doi.org/10.1016/S07351097(01)01326-2

7. Thambo JB, Bordachar P, Garrigues A, Lafitte S, Sanders P, Reuter $S$, et al. Detrimental ventricular remodeling in patients with congenital complete heart block and chronic right ventricular apical pacing. Circulation. 2004;110(25):3766-72. https://doi.org/10.1161/01.CIR.0000150336.86033.8D

8. Melo CS, Cardinalli Neto A, Silva LM. Histórico da estimulação cardíaca artificial. In: CS. Melo. Temas de marca-passo. São Paulo: Lemos Editorial. 2001, p. 19-44. 